\title{
Enclathration of $\mathrm{X} @ \mathrm{La}_{4}$ tetrahedra in channels of Zn-P frameworks in $\mathrm{La}_{3} \mathrm{Zn}_{4} \mathbf{P}_{6} \mathrm{X}(\mathrm{X}=\mathrm{Cl}, \mathrm{Br})$
}

Jian Wang $^{\dagger}$, Derrick Kaseman ${ }^{\ddagger}$, Kathleen Lee ${ }^{\dagger}$, Sabyasachi Sen ${ }^{\ddagger}$, Kirill Kovnir ${ }^{\dagger} *$

${ }^{\dagger}$ Department of Chemistry, and ${ }^{\ddagger}$ Department of Materials Science, University of California, Davis, One Shields Avenue, Davis, CA 95616, United States

\section{Table of Contents}

Figure S1. Powder X-ray diffraction patterns for $\mathrm{La}_{3} \mathrm{Zn}_{4} \mathrm{P}_{6} \mathrm{Cl}$ (top) and calculated diffraction patterns (bottom)

Figure S2. Powder X-ray diffraction patterns for $\mathrm{La}_{3} \mathrm{Zn}_{4} \mathrm{P}_{6.6} \mathrm{Br}_{0.8}$ (top) and calculated diffraction patterns (bottom).

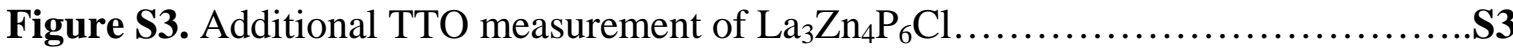

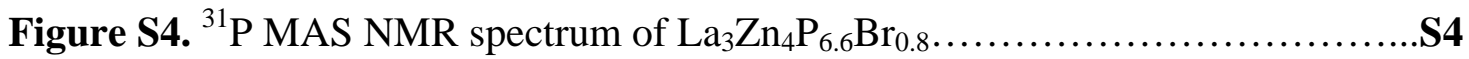

Table S1. Selected crystal data and structure refinement parameters

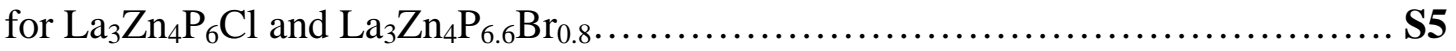

Table S2. Refined atomic coordinates and isotropic displacement parameters

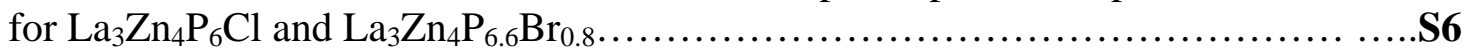

Table S3. Selected interatomic distances in $\mathrm{La}_{3} \mathrm{Zn}_{4} \mathrm{P}_{6} \mathrm{Cl}$ and $\mathrm{La}_{3} \mathrm{Zn}_{4} \mathrm{P}_{6.6} \mathrm{Br}_{0.8} \ldots \ldots . . . \mathrm{S7}$

Table S4. Parameters used in quantum chemical calculations. ... .58

Table S5. Energy-dispersive X-ray spectroscopy results of $\mathrm{La}_{3} \mathrm{Zn}_{4} \mathrm{P}_{6} \mathrm{Cl}$ and $\mathrm{La}_{3} \mathrm{Zn}_{4} \mathrm{P}_{6.6} \mathrm{Br}_{0.8}$

Figure S5. Crystal orbital Hamilton population plots for selected bonds in $\mathrm{La}_{3} \mathrm{Zn}_{4} \mathrm{P}_{6} \mathrm{Cl}$ and $\mathrm{La}_{3} \mathrm{Zn}_{4} \mathrm{P}_{6.6} \mathrm{Br}_{0.8}$

Figure S6. Thermal conductivities for $\left.\mathrm{La}_{3} \mathrm{Zn}_{4} \mathrm{P}_{6} \mathrm{Cl}\right)$ and $\mathrm{La}_{3} \mathrm{Zn}_{4} \mathrm{P}_{6.6} \mathrm{Br}_{0.8}$ 


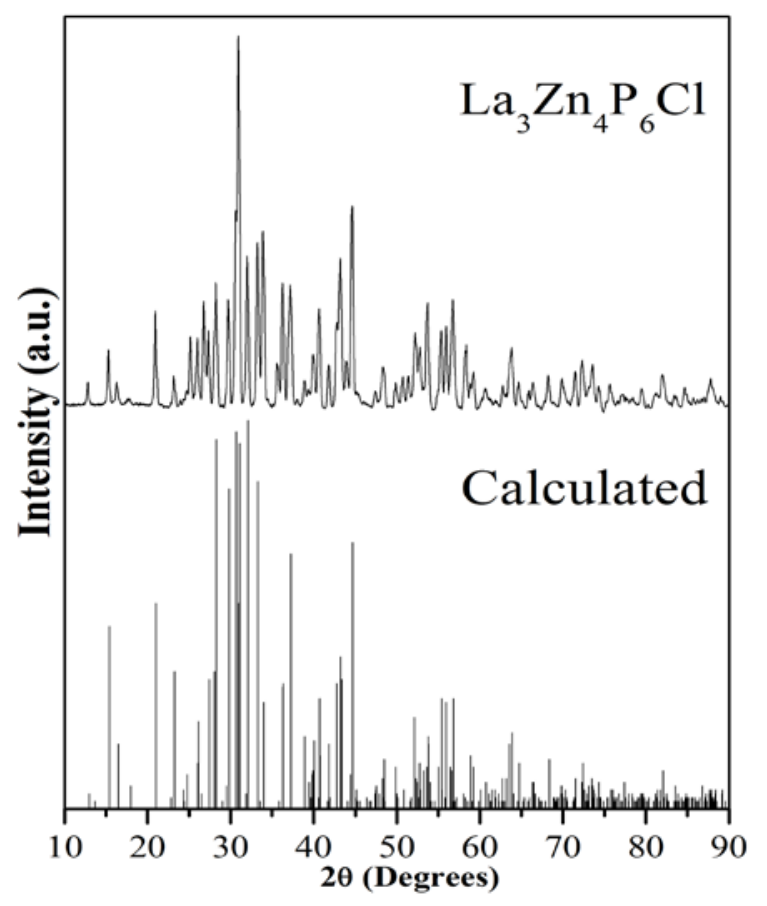

Figure S1. Powder X-ray diffraction patterns for $\mathrm{La}_{3} \mathrm{Zn}_{4} \mathrm{P}_{6} \mathrm{Cl}$ (top) and calculated diffraction patterns (bottom).

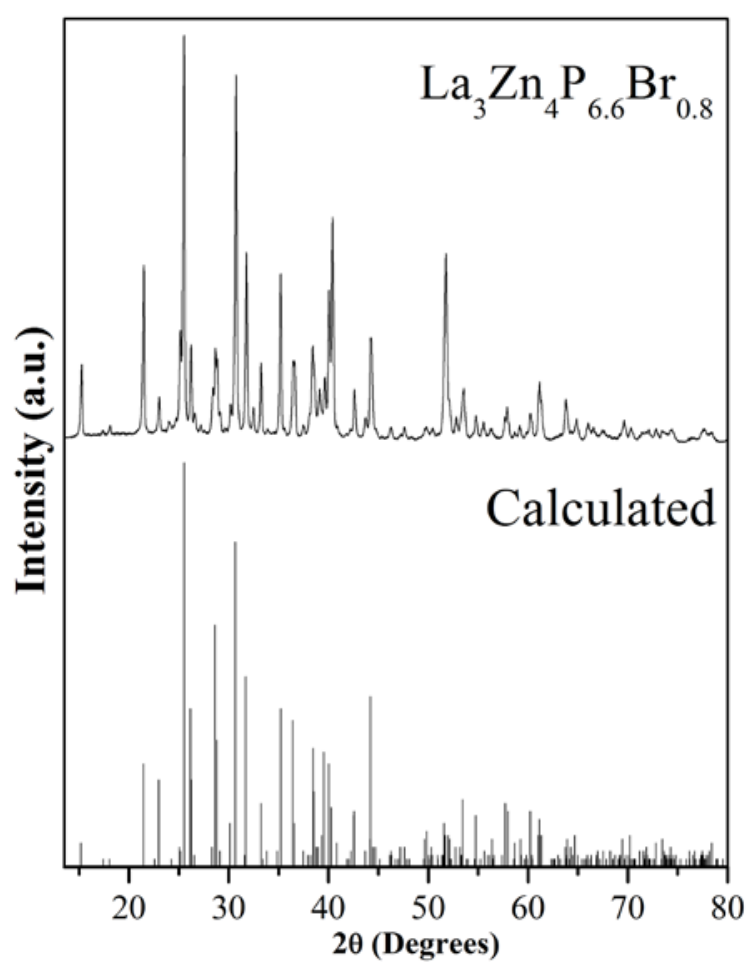

Figure S2. Powder $\mathrm{X}$-ray diffraction patterns for $\mathrm{La}_{3} \mathrm{Zn}_{4} \mathrm{P}_{6.6} \mathrm{Br}_{0.8}$ (top) and calculated diffraction patterns (bottom). 


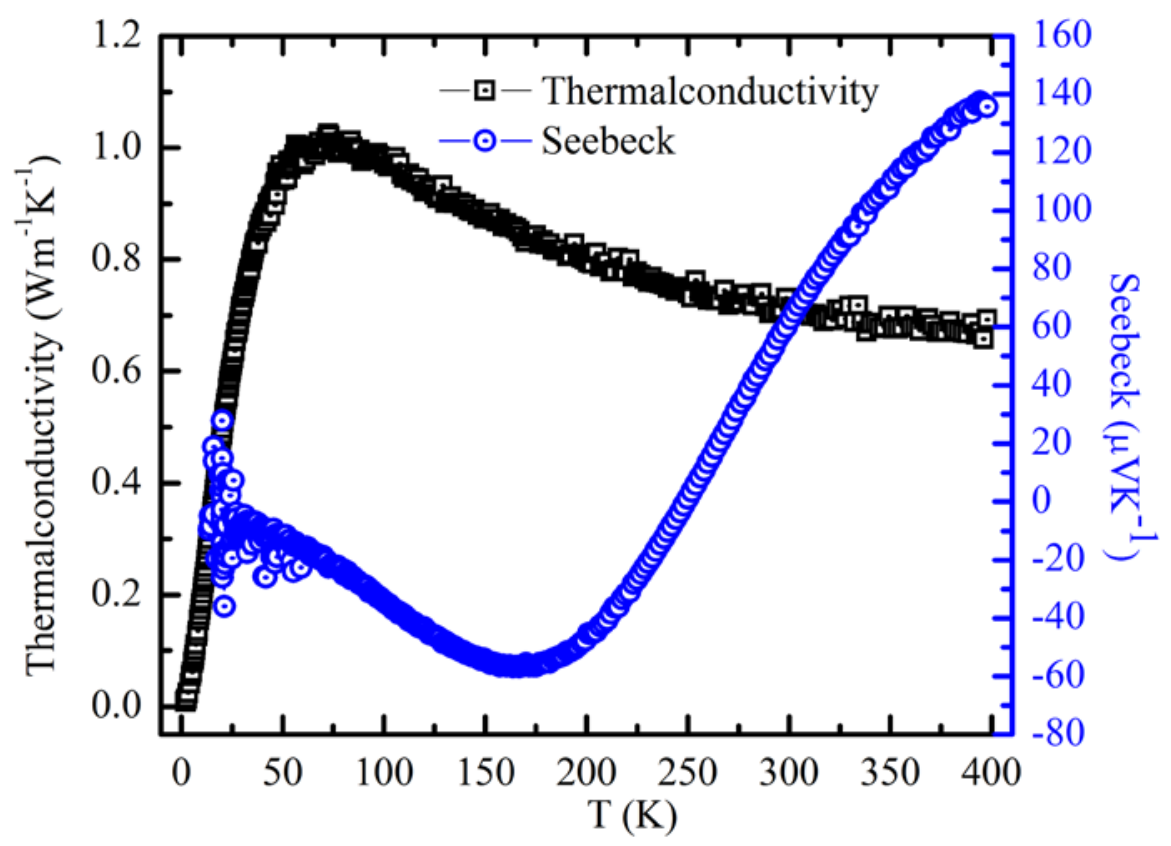

Figure S3. Additional TTO measurement of $\mathrm{La}_{3} \mathrm{Zn}_{4} \mathrm{P}_{6} \mathrm{Cl}$ showing good reproducibility. 

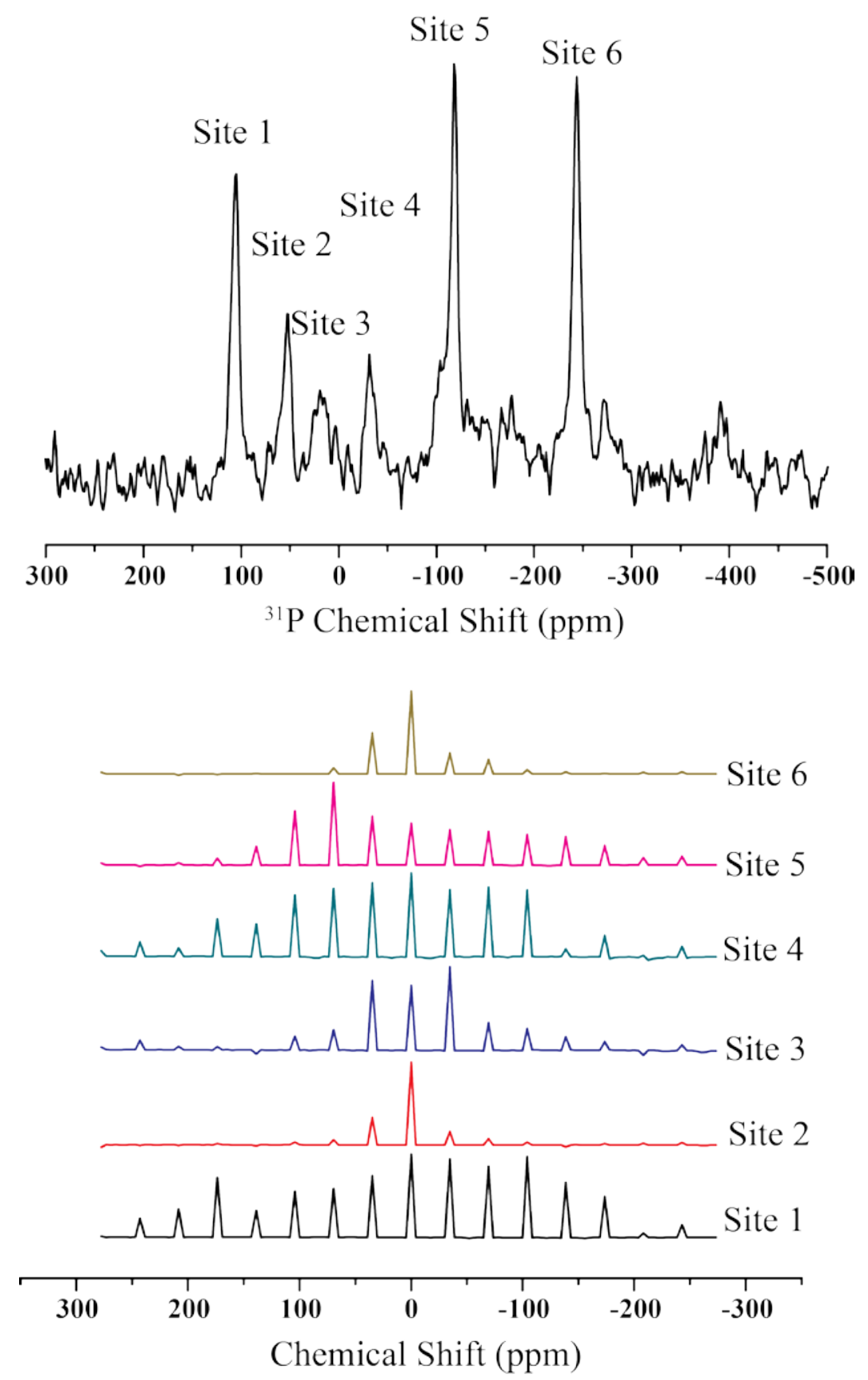

Figure S4. ${ }^{31} \mathrm{P}$ MAS NMR spectrum of $\mathrm{La}_{3} \mathrm{Zn}_{4} \mathrm{P}_{6.6} \mathrm{Br}_{0.8}$. Top: experimental spectrum collected at s spinning speed of $15 \mathrm{KHz}$; Bottom: Isotropic chemical shifts of sites 1 to 6 . 
Table S1. Selected crystal data and structure refinement parameters for $\mathrm{La}_{3} \mathrm{Zn}_{4} \mathrm{P}_{6} \mathrm{Cl}$ and $\mathrm{La}_{3} \mathrm{Zn}_{4} \mathrm{P}_{6.6} \mathrm{Br}_{0.8}$

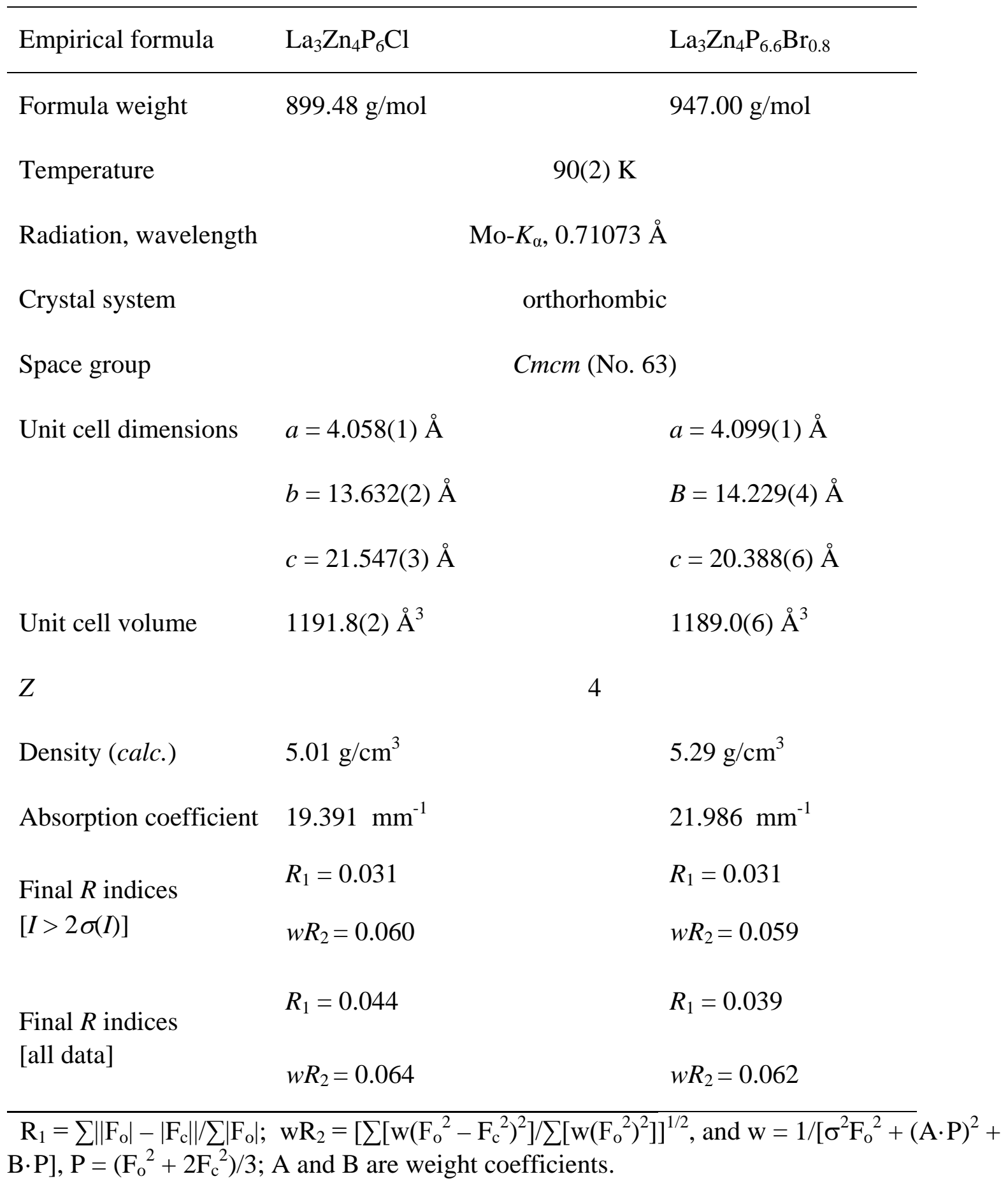


Table S2. Refined atomic coordinates and isotropic displacement parameters for $\mathrm{La}_{3} \mathrm{Zn}_{4} \mathrm{P}_{6} \mathrm{Cl}$ and $\mathrm{La}_{3} \mathrm{Zn}_{4} \mathrm{P}_{6.6} \mathrm{Br}_{0.8}$.

\begin{tabular}{|c|c|c|c|c|c|c|}
\hline Atoms & Wyckoff & $x / a$ & $y / b$ & $z / c$ & Occupancy & $U_{\text {eq }}\left(\AA^{2}\right)^{a}$ \\
\hline \multicolumn{7}{|c|}{$\mathrm{La}_{3} \mathrm{Zn}_{4} \mathrm{P}_{6} \mathrm{Cl}$} \\
\hline La1 & $8 f$ & 0 & $0.24176(4)$ & $0.62448(2)$ & 1 & $0.0055(1)$ \\
\hline La2 & $4 c$ & 0 & $0.50715(5)$ & $1 / 4$ & 1 & $0.0049(2)$ \\
\hline Zn1 & $8 f$ & 0 & $0.01805(7)$ & $0.11029(5)$ & 1 & $0.0073(2)$ \\
\hline $\mathrm{Zn} 2$ & $8 f$ & 0 & $0.36519(7)$ & $0.01400(5)$ & 1 & $0.0091(2)$ \\
\hline $\mathrm{P} 1$ & $8 f$ & 0 & $0.1436(2)$ & $0.1984(1)$ & 1 & $0.0062(4)$ \\
\hline $\mathrm{P} 2$ & $8 f$ & 0 & $0.1557(2)$ & $0.0414(1)$ & 1 & $0.0065(4)$ \\
\hline P3 & $8 f$ & 0 & $0.4215(2)$ & $0.1174(1)$ & 1 & $0.0057(4)$ \\
\hline $\mathrm{Cl} 1$ & $4 c$ & 0 & $0.8505(2)$ & $1 / 4$ & 1 & $0.0069(5)$ \\
\hline \multicolumn{7}{|c|}{$\mathrm{La}_{3} \mathrm{Zn}_{4} \mathbf{P}_{6.6} \mathrm{Br}_{0.8}$} \\
\hline La1 & $8 f$ & 0 & $0.28338(3)$ & $0.11201(2)$ & 1 & $0.0066(1)$ \\
\hline La2 & $4 c$ & 0 & $0.03976(5)$ & $1 / 4$ & 1 & $0.0067(2)$ \\
\hline Zn1 & $8 f$ & 0 & $0.09395(7)$ & $0.00941(5)$ & 1 & $0.0096(2)$ \\
\hline $\mathrm{Zn} 2$ & $8 f$ & 0 & $0.53950(7)$ & $0.11094(5)$ & 1 & $0.0090(2)$ \\
\hline $\mathrm{Br} 1$ & $4 c$ & 0 & $0.37789(9)$ & $1 / 4$ & $0.80(1)$ & $0.0068(4)$ \\
\hline $\mathrm{P} 1$ & $8 f$ & 0 & $0.6680(2)$ & $0.1943(1)$ & 1 & $0.0075(4)$ \\
\hline $\mathrm{P} 2$ & $8 f$ & 0 & $0.0520(2)$ & $0.6052(1)$ & 1 & $0.0073(4)$ \\
\hline P3 & $8 f$ & 0 & $0.3284(2)$ & $0.5362(1)$ & 1 & $0.0068(4)$ \\
\hline P4 & $4 c$ & 0 & $0.37789(9)$ & $1 / 4$ & 0.20 & $0.0068(4)$ \\
\hline P5 & $4 c$ & 0 & $0.8083(6)$ & $1 / 4$ & $0.41(2)$ & $0.012(2)$ \\
\hline
\end{tabular}

${ }^{a} U_{\text {eq }}$ is defined as one third of the trace of the orthogonalized $U^{i j}$ tensor 
Table S3. Selected interatomic distances $(\AA)$ in $\mathrm{La}_{3} \mathrm{Zn}_{4} \mathrm{P}_{6} \mathrm{Cl}$ and $\mathrm{La}_{3} \mathrm{Zn}_{4} \mathrm{P}_{6.6} \mathrm{Br}_{0.8}$.

\begin{tabular}{|c|c|c|c|}
\hline Atom pairs & Distances $(\AA)$ & Atom pairs & Distances $(\AA)$ \\
\hline \multicolumn{4}{|c|}{$\mathrm{La}_{3} \mathrm{Zn}_{4} \mathbf{P}_{6} \mathrm{Cl}$} \\
\hline La1- P1×2 & $3.016(2)$ & $\mathrm{P} 1-\quad \mathrm{P} 1$ & $2.221(4)$ \\
\hline $\mathrm{P} 2 \times 2$ & $3.046(2)$ & Zn1 & $2.557(2)$ \\
\hline P3 $\times 2$ & $3.015(2)$ & $\mathrm{La} 1 \times 2$ & $3.016(2)$ \\
\hline $\mathrm{Cl} 1$ & 2.983(1) & $\mathrm{La} 2 \times 2$ & $2.968(2)$ \\
\hline La2- P1×4 & $2.968(2)$ & $\mathrm{P} 2-\quad \mathrm{Zn} 1$ & $2.392(2)$ \\
\hline $\mathrm{P} 3 \times 2$ & $3.086(2)$ & $\mathrm{Zn} 2 \times 2$ & $2.371(1)$ \\
\hline $\mathrm{Cl} 1 \times 2$ & $2.946(2)$ & $\mathrm{Zn} 2$ & $2.916(3)$ \\
\hline \multirow{2}{*}{$\begin{array}{r}\mathrm{Zn} 1-\quad \mathrm{P} 1 \\
\text { P2 }\end{array}$} & $2.557(2)$ & $\mathrm{La} 1 \times 2$ & $3.046(2)$ \\
\hline & $2.392(2)$ & P3 $-\mathrm{Zn} 1 \times 2$ & $2.423(1)$ \\
\hline \multirow{2}{*}{$\begin{array}{r}\mathrm{P} 3 \times 2 \\
\mathrm{Zn} 2-\mathrm{P} 2 \times 2\end{array}$} & $2.423(1)$ & Zn2 & $2.356(2)$ \\
\hline & $2.371(1)$ & $\mathrm{La} 1 \times 2$ & $3.015(2)$ \\
\hline P2 & $2.916(2)$ & La2 & $3.086(2)$ \\
\hline \multirow[t]{2}{*}{ P3 } & $2.356(2)$ & $\mathrm{Cl} 1-\mathrm{La} 1 \times 2$ & $2.983(1)$ \\
\hline & & $\mathrm{La} 2 \times 2$ & $2.946(2)$ \\
\hline \multicolumn{4}{|c|}{$\mathbf{L a}_{3} \mathrm{Zn}_{4} \mathbf{P}_{6.6} \mathrm{Br}_{0.8}$} \\
\hline $\mathrm{La} 1-\mathrm{P} 1 \times 2$ & $3.116(2)$ & $\mathrm{P} 1-\mathrm{P} 1$ & $2.272(4)$ \\
\hline $\mathrm{P} 2 \times 2$ & $3.115(2)$ & P5 & $2.297(8)$ \\
\hline P3 & $3.088(2)$ & Zn2 & $2.496(2)$ \\
\hline $\mathrm{P} 3 \times 2$ & $3.020(2)$ & $\mathrm{La} 1 \times 2$ & $3.116(2)$ \\
\hline $\mathrm{Br} 1 / \mathrm{P} 4$ & $3.118(1)$ & $\mathrm{La} 2 \times 2$ & $2.970(2)$ \\
\hline $\mathrm{La} 2-\mathrm{P} 1 \times 4$ & $2.970(2)$ & $\mathrm{P} 2-\mathrm{Zn} 1$ & $2.411(2)$ \\
\hline $\mathrm{P} 2 \times 2$ & $3.228(2)$ & $\mathrm{Zn} 1$ & $2.851(2)$ \\
\hline $\mathrm{Br} 1 / \mathrm{P} 4 \times 2$ & $3.083(1)$ & $\mathrm{Zn} 2 \times 2$ & $2.431(1)$ \\
\hline P5 & 3.293(9) & $\mathrm{La} 1 \times 2$ & $3.115(2)$ \\
\hline Zn1- P2 & $2.411(2)$ & La2 & $3.228(2)$ \\
\hline
\end{tabular}




$\begin{array}{rllcl} & \text { P2 } & 2.851(2) & \mathrm{P} 3-\mathrm{Zn} 1 \times 2 & 2.392(1) \\ \mathrm{Zn} 2- & \mathrm{P} 3 \times 2 & 2.392(1) & \mathrm{Zn} 2 & 2.421(2) \\ \mathrm{P} 1 & 2.496(2) & \mathrm{La} 1 \times 2 & 3.020(2) \\ \mathrm{P} 2 \times 2 & 2.431(1) & \mathrm{La} 1 & 3.088(2) \\ \mathrm{P} 3 & 2.421(2) & \mathrm{P} 5-\mathrm{P} 1 \times 2 & 2.297(8) \\ \mathrm{Br} 1 / \mathrm{P} 4-\mathrm{P} 5 \times 2 & 2.276(4) & \mathrm{Br} 1 / \mathrm{P} 4 \times 2 & 2.276(4) \\ \mathrm{La} 1 \times 2 & 3.118(1) & \mathrm{La} 2 & 3.293(9) \\ \mathrm{La} 2 \times 2 & 3.083(1) & & \end{array}$

Table S4. Parameters used in quantum chemical calculations.

\begin{tabular}{|l|l|l|}
\hline Compound/Model & $\mathrm{La}_{3} \mathrm{Zn}_{4} \mathrm{P}_{6} \mathrm{Cl}$ & $\mathrm{La}_{3} \mathrm{Zn}_{4} \mathrm{P}_{6} \mathrm{Br} \& \mathrm{La}_{3} \mathrm{Zn}_{4} \mathrm{P}_{7} \mathrm{Br}$ \\
\hline$k$-mesh & $16 \times 16 \times 8$ & $16 \times 16 \times 8$ \\
\hline Irreducible $k$-points & 365 & 365 \\
\hline Basis set & $\mathrm{La}(6 s, 5 d, 4 f)$ & $\mathrm{La}(6 s, 5 d, 4 f)$ \\
& $\mathrm{Zn}(4 s, 4 p, 3 d)$ & $\mathrm{Zn}(4 s, 4 p, 3 d)$ \\
& $\mathrm{P}(3 s, 3 p)$ & $\mathrm{P}(3 s, 3 p)$ \\
& $\mathrm{Cl}(3 p)$ & $\mathrm{Br}(4 p)$ \\
\hline Downfolded orbitals & $\mathrm{La}(6 p), \mathrm{P}(3 d)$ & $\mathrm{La}(6 p), \mathrm{P}(3 d)$ \\
& $\mathrm{Cl}(4 s, 3 d)$ & $\mathrm{Br}(5 s, 4 d)$ \\
& &
\end{tabular}


Table S5. Energy-dispersive X-ray spectroscopy results of $\mathrm{La}_{3} \mathrm{Zn}_{4} \mathrm{P}_{6} \mathrm{Cl}$.

\begin{tabular}{|l|l|l|}
\hline & Composition \\
\hline & $\mathrm{La}_{3.04} \mathrm{Zn}_{4} \mathrm{P}_{5.84} \mathrm{Cl}_{1.07}$ & \\
\hline \\
\hline
\end{tabular}

Average: $\mathbf{L a}_{3.0(1)} \mathbf{Z n}_{4} \mathbf{P}_{6.0(1)} \mathbf{C l}_{1.1(3)}$ 


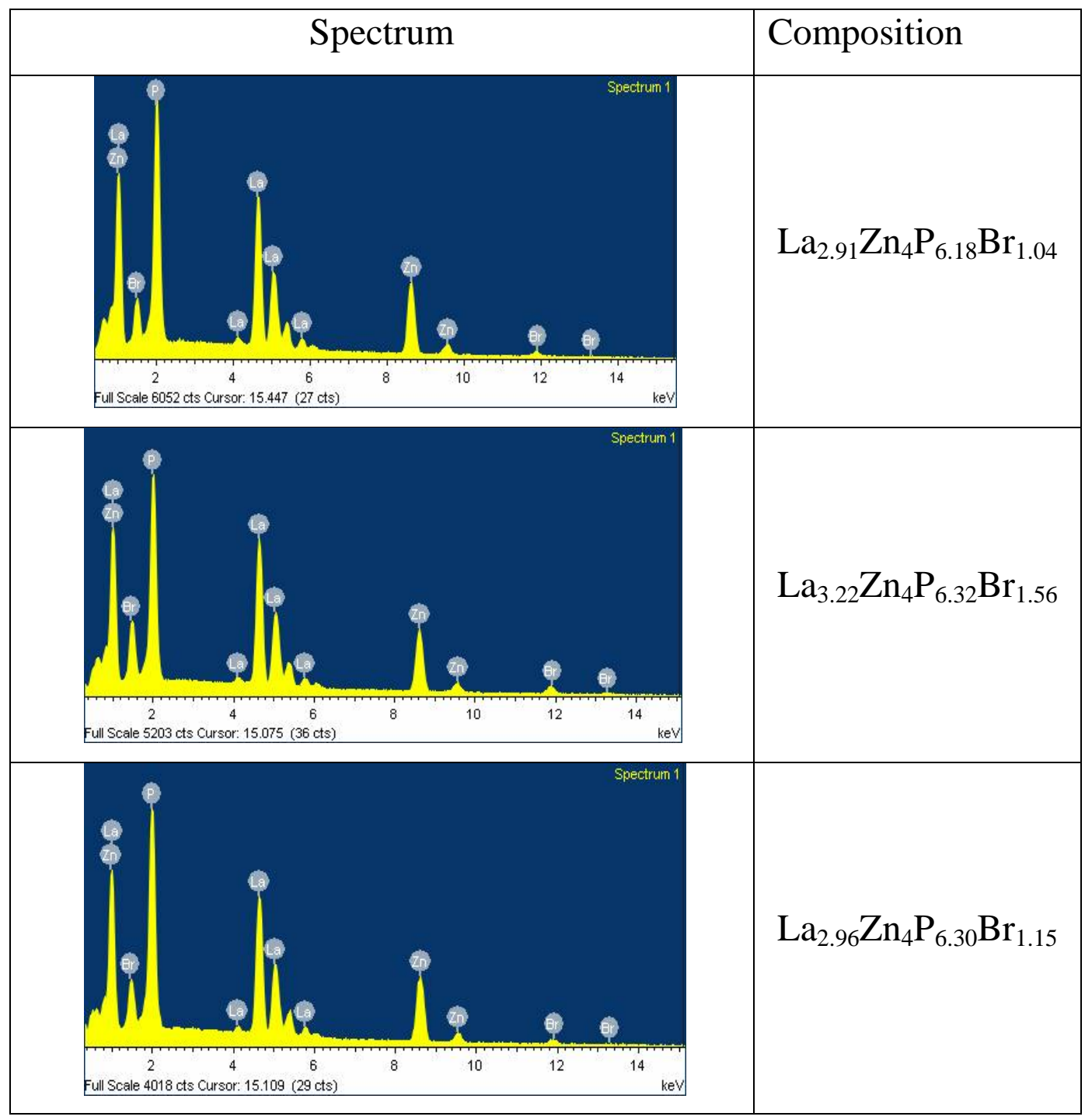

Average: $\mathbf{L a}_{3.0(2)} \mathbf{Z n}_{4} \mathbf{P}_{6.3(1)} \mathbf{B r}_{1.2(3)}$ 


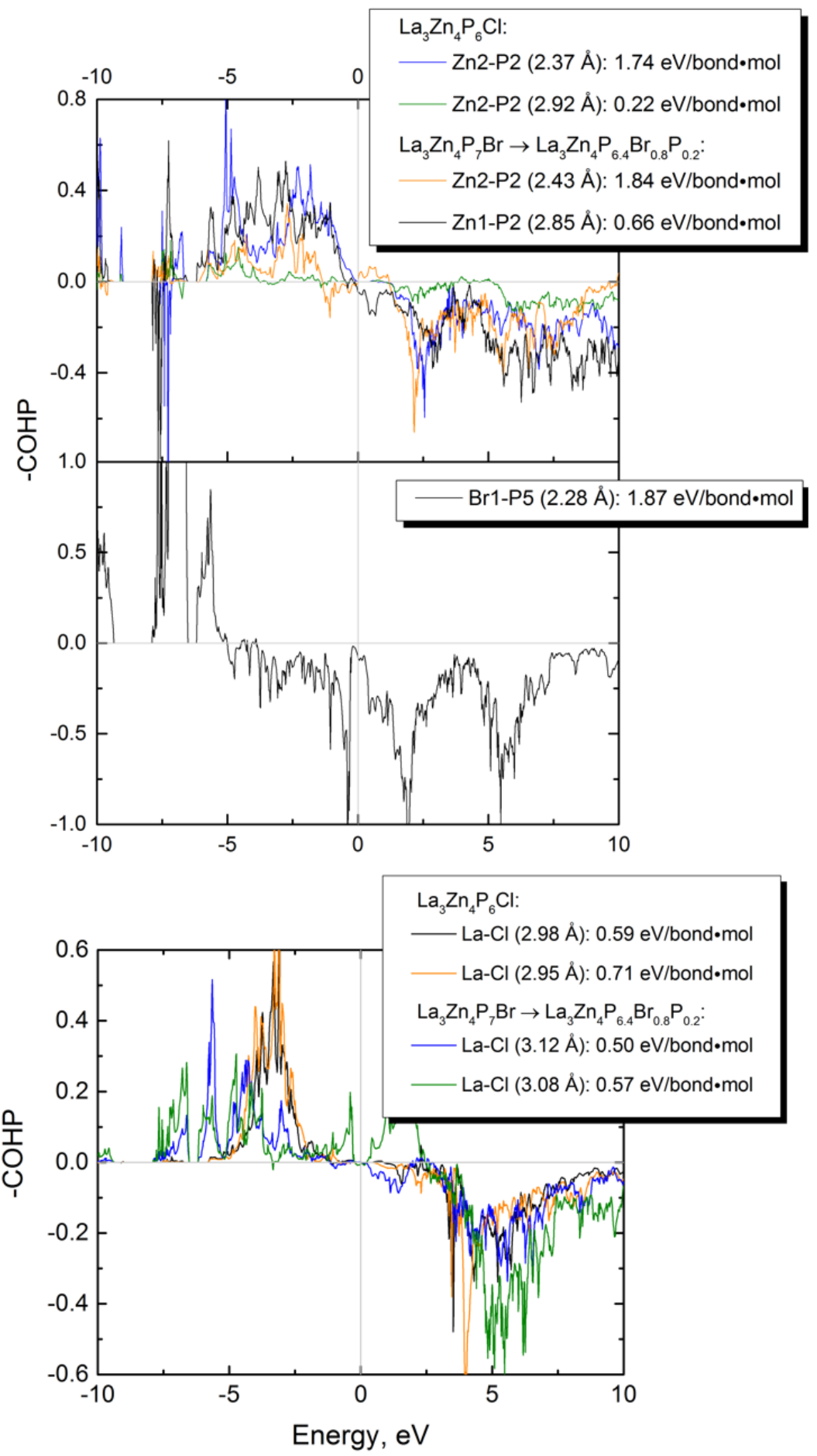

Figure S5. Crystal orbital Hamilton population plots for selected bonds. The Fermi level was adjusted for $\mathrm{La}_{3} \mathrm{Zn}_{4} \mathrm{P} 7 \mathrm{Br}$ to the correct composition, $\mathrm{La}_{3} \mathrm{Zn}_{4} \mathrm{P}_{6.6} \mathrm{Br}_{0.8}$. $-\mathrm{ICOHP}$ values are given in the legends. 

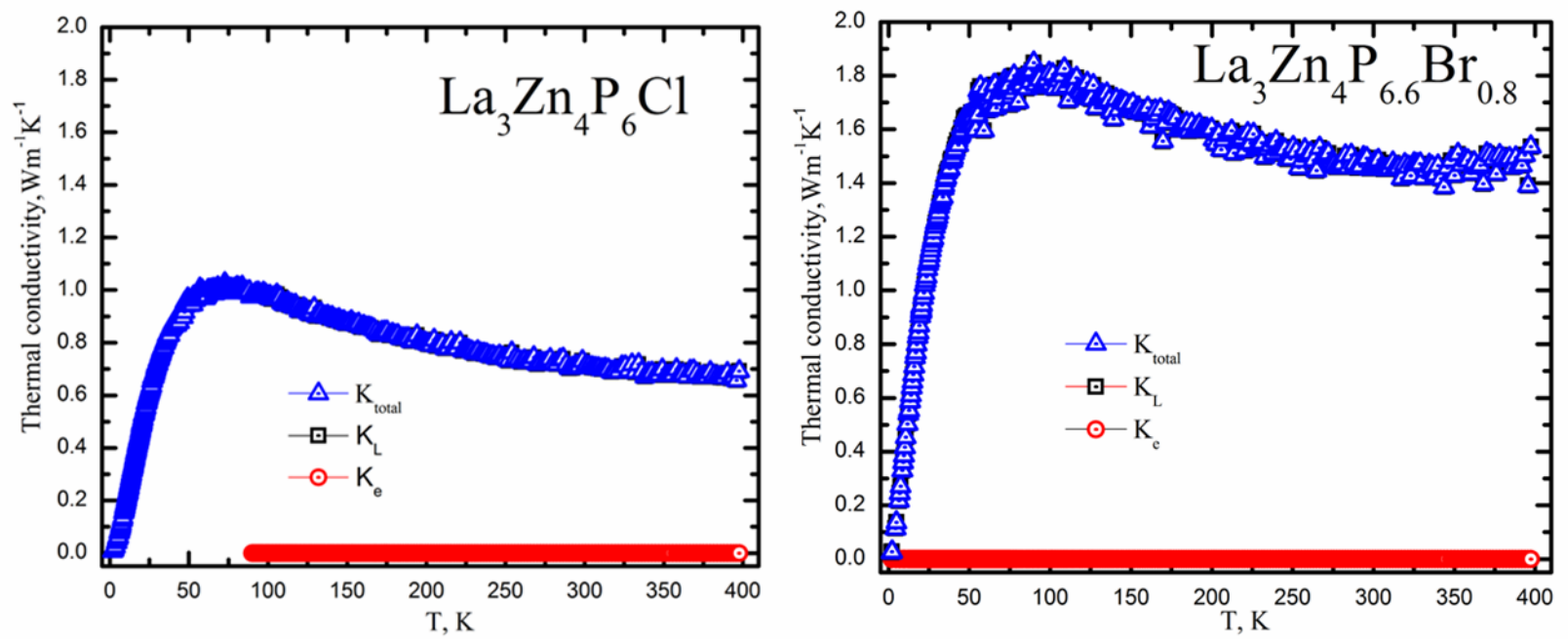

Figure S6. Temperature dependences of the thermal conductivity with lattice (black) and electronic (red) contributions for $\mathrm{La}_{3} \mathrm{Zn}_{4} \mathrm{P}_{6} \mathrm{Cl}$ (left) and $\mathrm{La}_{3} \mathrm{Zn}_{4} \mathrm{P}_{6.6} \mathrm{Br}_{0.8}$ (right). Note that due to extremely small electronic contribution, black and blue symbols overlap. 